Itinéraires Itinéraires

Littérature, textes, cultures

\title{
Traditions et modernités : Eliot face à la temporalité française
}

William Marx

\section{(2) OpenEdition}

\section{Journals}

Édition électronique

URL : http://journals.openedition.org/itineraires/475

DOI : 10.4000/itineraires.475

ISSN : 2427-920X

Éditeur

Pléiade

\section{Édition imprimée}

Date de publication : 1 novembre 2009

Pagination : 77-88

ISBN : 978-2-296-10115-9

ISSN : 2100-1340

\section{Référence électronique}

William Marx, "Traditions et modernités : Eliot face à la temporalité française », Itinéraires [En ligne] 2009-3 | 2009, mis en ligne le 24 juin 2014, consulté le 03 mai 2019. URL : http://

journals.openedition.org/itineraires/475; DOI : 10.4000/itineraires.475

\section{(ब) $(\Theta \Theta$}

Itinéraires est mis à disposition selon les termes de la licence Creative Commons Attribution - Pas d'Utilisation Commerciale - Pas de Modification 4.0 International. 


\title{
Traditions et modernités : Eliot face à la temporalité française
}

\begin{abstract}
Why is the concept of modernism so unusual in French literature, unlike English and American literature? Why is the concept of classicism so usual in French literature, but not in Anglo-American literature? Our hypothesis is that both questions are connected: there are two different modernisms because the two literatures have radically different visions of time and of their own past. We try to prove that hypothesis through a study of T. S. Eliot's and The Criterion's complicated and ambiguous relationship with $L a$ Nouvelle Revue française.
\end{abstract}

Keywords : T. S. Eliot, La Nouvelle Revue française, The Criterion, modernism, classicism

Mots clés : T. S. Eliot, La Nouvelle Revue française, The Criterion, modernisme, classicisme

Si le modernisme suppose un certain rapport à l'histoire, au présent de l'action, à un futur projeté et, symétriquement, à un passé rejeté ou choisi, pourquoi penser que cette expérience du temps serait semblable dans tous les pays ou toutes les cultures? Or, s'il y a pluralité des expériences temporelles et des conceptions de l'histoire, celle-ci implique forcément une pluralité du modernisme.

Qu'on songe tout d'abord à l'imprécision des limites temporelles assignées à ce mouvement. Commença-t-il dès les années 1890? Se poursuivit-il jusque dans les années 1940? Faut-il distinguer, à la suite de Frank Kermode, entre un «paléomodernisme » (avant la Première Guerre mondiale) et un " néomodernisme » (pendant l'entre-deux-guerres ${ }^{1}$ ) ? Ce flou temporel de la notion peut être corrélé à un autre flou, de caractère géographique et culturel: employé extensivement dans le monde

1. Voir Frank Kermode, Modern Essays, Londres, Collins, 1971, p. 46 (« The Modern »). 
anglophone pour désigner grosso modo un mouvement d'innovation esthétique couvrant tous les arts et rassemblant, en littérature, des figures telles que James, Conrad, Joyce, Woolf, Faulkner, Yeats, Pound et Eliot, pour ne citer que les noms les plus célèbres, le terme est très peu utilisé dans les autres littératures (en mettant à part le modernismo de Rubén Darío). Le fait est suffisamment surprenant pour devoir être signalé et analysé.

En France, par exemple, on parle plutôt de surréalisme ou d'avantgardes, mais ces mots ont une acception évidemment beaucoup plus étroite que celle de modernisme. La différence est d'abord d'ordre chronologique : habituellement, les histoires de la littérature française font commencer le $\mathrm{XX}^{\mathrm{e}}$ siècle véritable à la Première Guerre mondiale, c'est-à-dire à Dada et au surréalisme, qui marquent selon elles une authentique rupture avec le passé, de sorte que la première décennie du siècle est jetée aux oubliettes. À l'inverse des Anglo-Américains, les Français éprouvent beaucoup de mal à envisager comme un tout la période allant des années 1890 aux années 1940. Cette absence de coïncidence entre la périodisation de la littérature française et celle de la littérature anglophone rend difficile le dialogue entre les historiens : non seulement la notion de modernisme n'est pas comprise des Français, mais encore il n'est pas aisé de lui trouver une traduction. Par exemple, un critique anglophone décrira sans état d'âme André Gide et Paul Valéry comme des écrivains modernistes, alors qu'un critique français répugnerait à les ranger dans ce qui pourrait lui apparaître comme l'équivalent du modernisme, c'est-à-dire les avant-gardes, du moins celles des années 1920, qu'il ne saurait confondre avec celles des années 1890.

Outre cette divergence des périodisations historiques, l'incompréhension entre les deux critiques est accentuée par les connotations radicalement différentes que chacun attache à la notion de modernisme. Sur le modèle du programme surréaliste, le Français comprend le projet moderniste comme une volonté de faire table rase du passé, alors que dans le modernisme anglophone, on constate une bien plus grande ambivalence du rapport à l'histoire : plutôt que de rejeter le passé d'un seul bloc, les écrivains angloaméricains préfèrent opérer sur lui une sorte de sélection pour le reconfigurer à la lumière des exigences du présent. Quand André Breton se présentait comme un terroriste et un révolutionnaire de l'esthétique, de son côté Eliot proposait à l'apprenti-écrivain de s'absorber d'abord avec humilité dans la tradition de son pays s'il voulait trouver l'originalité véritable ${ }^{2}$. Dans cette opposition emblématique de deux écrivains phares de l'avant-garde, on retrouve la double vision de l'histoire qui détermine profondément le sens attaché au concept de modernisme de part et d'autre de la Manche, voire de l'Atlantique. C'est en fait à deux types de modernismes que l'on a affaire, dans une ambiguïté qui peut être source de nombreux malentendus

2. Voir Thomas Stearns Eliot, Selected Essays, Londres, Faber and Faber, 1951, p. 13-22 (« Tradition and the Individual Talent », 1919). 
et qu'on ne pourra lever qu'en s'interrogeant sur la base même du projet moderniste, c'est-à-dire sur la question tellement problématique de la relation du présent artistique avec le passé ${ }^{3}$. Dans la mesure où la pensée du progrès dans les arts est inséparable d'une pensée du passé, on verra que les noms par lesquels chaque culture a l'habitude de désigner ce passé ne sont pas anodins. Classique, traditionnel, canonique : ces mots, qui semblent a priori synonymes, le sont si peu en réalité qu'ils déterminent des options esthétiques fondamentales, exclusives l'une de l'autre. Là réside peut-être la différence principale entre les deux modernismes, qui relève d'un écart fondamental entre la culture française et la culture anglophone.

Pour mettre à l'épreuve cette hypothèse, on proposera ici une sorte de test grandeur nature des relations entre les deux littératures, en étudiant l'exemple de l'un des malentendus historiques les plus significatifs : celui qui fit se confronter, à travers la figure centrale de T. S. Eliot, les deux revues littéraires les plus emblématiques du modernisme français (au sens anglophone du terme) et du modernisme britannique, La Nouvelle Revue française d'un côté, The Criterion de l'autre. À l'évidence, Eliot occupe une position éminente dans les relations que La Nouvelle Revue française entretint avec le modernisme anglophone, puisqu'il fut tout à la fois un collaborateur de la revue et une icône majeure de la littérature angloaméricaine de l'entre-deux-guerres. Dans la collaboration d'Eliot à la $N R F$, on pourrait donc trouver a priori la preuve d'une certaine communauté de vues entre les deux littératures. Mais en fait on verra que la situation n'est pas si simple et que les liens qui unirent l'écrivain anglo-américain à la revue étaient tissés d'ambiguïtés, révélatrices d'un quiproquo culturel capital. Sur fond d'une collaboration apparemment sans histoire, ce furent deux conceptions du modernisme qui s'affrontèrent - et deux conceptions du temps.

\section{La collaboration d'Eliot à la $N R F$}

Les relations d'Eliot avec la $N R F$ commencèrent par une rencontre manquée. Il se trouve que, lors de son premier séjour à Paris, de l'automne 1910 à l'été 1911, le jeune Américain, alors étudiant de Harvard, prit des leçons de conversation auprès d'Alain-Fournier, avec qui se noua une amitié $^{4}$. Un jour, il fut conduit par Alain-Fournier chez son beau-frère,

3. Voir William Marx (dir.), Les Arrière-gardes au XXe siècle : l'autre face de la modernité esthétique, Paris, PUF, 2004 ; rééd. 2008.

4. Voir Alain-Fournier, lettre à T. S. Eliot du 25 juillet 1911, dans Lettres à sa famille et à quelques autres, Paris, Fayard, 1991, p. 680-682. Commentaire par Stuart Barr, « À propos d'une lettre retrouvée d'Alain-Fournier à T. S. Eliot », Bulletin des amis de Jacques Rivière et d'Alain-Fournier, $\mathrm{n}^{\circ}$ 39, $1^{\text {er }}$ trimestre 1986, p. 7-18. Dans son livre sur Alain-Fournier, The Land without a Name: Fournier and his world (Londres, Elek, 1975), Robert Gibson raconte les souvenirs qu'Eliot lui avait confiés en 1951. 
Jacques Rivière, alors secrétaire de la $N R F$, afin de lui demander conseil sur un travail universitaire dans lequel il souhaitait se lancer ${ }^{5}$. Cette foislà, le contact n'alla pas plus loin.

Malgré sa rencontre de 1911 avec Rivière, c'est en fait à André Gide qu'Eliot dut de devenir un collaborateur de la $N R F$. Il s'agissait pour Gide, en 1921, de trouver un successeur au titulaire de la rubrique « Lettre d'Angleterre », le critique jamesien Percy Lubbock, qui n'avait pas donné satisfaction: Gide lui reprochait de ne pas rendre compte des ouvrages de Lytton Strachey et de dresser ipso facto contre la $N R F$ les écrivains anglais les plus intéressants du moment, autrement dit le groupe de Bloomsbury. Consulté, Strachey recommanda Eliot avec d'autant plus de chaleur qu'il connaissait les déboires financiers, conjugaux et psychologiques traversés alors par le jeune poète ${ }^{6}$. Gide écrivit aussitôt à Eliot $^{7}$, et c'est ainsi que s'engagea une collaboration qui paraissait prometteuse.

En réalité, pendant les six années que dura cette collaboration, Eliot ne publia en moyenne qu'un article par an ${ }^{8}$. Et sur les six articles, deux parurent dans des numéros spéciaux d'hommage à Rivière en 1925 et à Mallarmé en 1926. Seuls quatre articles relevèrent effectivement de la rubrique "Lettre d'Angleterre». Pourquoi une telle parcimonie? Eliot n'aurait pu se plaindre d'avoir été maltraité par la $N R F$, puisque cette dernière plaça systématiquement son texte en première position parmi les contributions étrangères. Malheureusement, le début de sa collaboration coïncida avec la fondation de la revue The Criterion, laquelle commença désormais d'occuper une grande partie de son temps. Et surtout Eliot avait d'emblée prévenu Gide : «S'il s'agit seulement de livres de quelque valeur littéraire, je ne crois pas qu'il y ait de quoi rédiger un article tous les mois ${ }^{9}$. » Ainsi plaçait-il d'abord l'intérêt de la fonction de chroniqueur

5. Voir T. S. Eliot, «Rencontre », La Nouvelle Revue française, $n^{\circ} 139$, avril 1925, p. 657.

6. Voir André Gide et Jacques Rivière, Correspondance (1909-1925), Paris, Gallimard, 1998, p. 666, 668, 682-683, 685 (lettres à Rivière du 23 juillet, 14 août et 6 décembre 1921, et du 9 janvier 1922).

7. T. S. Eliot, Letters, vol. 1 : 1898-1922, Londres, Faber and Faber, 1988, p. 490-491 (lettre de Gide à Eliot du 7 décembre 1921).

8. T. S. Eliot, «Lettre d'Angleterre », La Nouvelle Revue française, ${ }^{\circ}{ }^{104}$, mai 1922 , p. 617-624; «Lettre d'Angleterre : le style dans la prose anglaise contemporaine », $\mathrm{n}^{\circ} 111$, décembre 1922, p. 751-756; «Lettre d'Angleterre », n 122, novembre 1923, p. 619-625; «Rencontre », $\mathrm{n}^{\circ} 139$, avril 1925, p. 657-658; « Note sur Mallarmé et Poe », n 158, novembre 1926, p. 524-526; "Les lettres anglaises : le roman contemporain », ${ }^{\circ} 164$, mai 1927, p. 669-675. Par ailleurs, en 1965, en hommage au poète qui venait de disparaître, la NRF publia la traduction, par Pierre Leyris, de The Cultivation of Christmas Trees. En 1947, Gide avait contribué à faire décerner un prix à la traduction par Leyris des Poèmes (1910-1930) d'Eliot (André Gide et Jean Schlumberger, Correspondance (1901-1950), Paris, Gallimard, p. 998).

9. T. S. Eliot, Letters, op. cit., p. 494 (lettre d'Eliot à Gide du 14 décembre 1921). 
dans son pouvoir de discrimination, voire d'exclusion, ce qui ne promettait pas une collaboration fort régulière.

De fait, la première « Lettre d'Angleterre » se montra, paradoxalement, fort négative à l'égard de la littérature anglaise, seules les œuvres écrites par des Irlandais (Wilde, Yeats et surtout Joyce) échappant à peu près au mépris dont Eliot accablait ses confrères. Dans cette première contribution, table rase était faite de plusieurs écrivains britanniques contemporains connus des lecteurs français : Wells, Bennett et surtout Kipling, qualifié de « pompier ». Une fois la place nette, il devenait possible de reconfigurer le canon de la littérature anglaise : c'est à quoi s'employa Eliot quelques mois après dans la chronique suivante. Il y tâcha de discerner l'émergence d'une nouvelle «tradition » d'écriture succédant à celle qu'avait lancée Pater : Joyce et Wyndham Lewis apparaissaient comme les germes d'un éventuel renouveau, parmi d'autres noms cités, tels que Virgina Woolf et D. H. Lawrence. Ainsi que l'avait espéré Gide, le nouveau chroniqueur des lettres anglaises accordait donc une place de choix à l'avant-garde moderniste, à la différence de son prédécesseur.

On remarque avec quel sérieux Eliot envisageait sa fonction de « correspondant anglais » de La Nouvelle Revue française ${ }^{10}$ : il ne concevait pas sa contribution comme un simple compte rendu périodique de l'actualité littéraire d'outre-Manche, mais comme un instrument pédagogique destiné à créer une nouvelle vision, hiérarchisée, architecturée, de la littérature anglaise contemporaine. Loin de se suivre au hasard, les articles d'Eliot s'organisaient selon une logique interne aisément perceptible.

Cet objectif allait devenir encore plus sensible dans la chronique suivante. Prenant davantage de recul par rapport à l'actualité et s'inspirant de l'Enquête sur les maîtres de la jeune littérature récemment parue en France ${ }^{11}$, Eliot s'efforça de mettre en évidence trois «maîtres » de la jeune littérature anglaise, cette fois-ci : Henry James, James Frazer et Francis Herbert Bradley ${ }^{12}$. Le propos visait plus que jamais à dérouler toute une généalogie en arrière-plan de la littérature contemporaine. La vision invoquée était de nature historique : il s'agissait, en récrivant le canon, de se réclamer d'une tradition encore latente.

Il est d'autant plus surprenant de voir, quatre années plus tard, en 1927, la dernière chronique d'Eliot adopter un ton radicalement différent,

10. Ibid., p. 510.

11. L'Enquête, conduite par Henri Rambaud et Pierre Varillon, venait d'être commentée par Benjamin Crémieux, "Le bilan d'une enquête ", La Nouvelle Revue française, ${ }^{\circ}$ 120, septembre 1923, p. 287-294.

12. Le nom du philosophe Bradley détonne un peu dans la triade : correspondant plutôt aux affinités personnelles d'Eliot qu'à une influence réelle sur l'ensemble de la jeune littérature, il satisfaisait d'abord au besoin de trouver à tout prix un pendant britannique à la figure de Bergson. 
bien plus critique à l'égard de la jeune génération. La psychologie des romans de Lawrence, Woolf, Garnett et Huxley y était condamnée au motif de leur désintérêt pour la question morale, tant et si bien qu'Eliot jugeait indignes d'appartenir à la postérité de James les mêmes écrivains dont il avait fait l'éloge en 1922. Les critiques adressées à l'esprit moderne annonçaient des essais beaucoup plus tardifs, comme After Strange Gods (1934). Le retournement par rapport aux premières chroniques semble donc total. Il faut, bien sûr, le mettre en rapport avec la conversion du poète à l'anglicanisme, qui eut lieu cette même année 1927.

Est-ce un hasard s'il s'agit du dernier texte publié dans la $N R F$ ? Pourtant, sa présentation laissait présager une collaboration d'Eliot plus soutenue : l'article n'apparaissait plus dans la section « Notes » comme d'habitude, mais avait été avancé dans la partie "Chroniques ", au même niveau qu'Alain et Thibaudet; son titre n'était plus « Lettre d'Angleterre », mais «Les lettres anglaises ». Si cette contribution n'eut pas de suite, il faut donc en chercher la cause moins du côté de la $N R F$ que d'Eliot luimême. Faute d'accès à la correspondance de l'écrivain dans ces années-là, l'hypothèse qu'on se contentera de formuler pour l'instant est celle-ci : le renversement critique qui eut lieu dans la chronique de 1927 creusa ou manifesta soudain un écart entre la position d'Eliot et celle de la $N R F$, compromettant toute collaboration ultérieure. De quelle nature fut cet écart? C'est ce qu'il s'agit maintenant de découvrir.

\section{The Criterion et la NRF}

En fait, la rupture d'Eliot avec la $N R F$ ou, du moins, son silence avaient été annoncés quelques années auparavant dans sa propre revue, The Criterion. Certes, à la fondation du Criterion en 1922, la $N R F$, visiblement considérée comme la revue littéraire de référence, avait servi de modèle, et son influence se manifestait dans maintes pages de la revue anglaise. Les premiers sommaires réservaient une place considérable non seulement aux maîtres canoniques de la jeune littérature française, Balzac, Flaubert, Mallarmé, mais encore aux auteurs les plus centraux de la $N R F$ : Larbaud, Proust, Rivière, Valéry et, plus tard, Fernandez et Benda. Le nombre élevé de reprises par le Criterion de textes précédemment parus dans la $N R F$ conférait presque à la revue d'Eliot le statut de simple filiale de la revue de Gide et de Rivière. Ce cousinage fut d'ailleurs quasiment officialisé en 1929, quand fut organisé un concours européen de la nouvelle regroupant cinq revues de cinq pays différents, dont The Criterion et la $N R F^{13}$.

Pourtant, dès le milieu des années 1920, la francophilie enthousiaste des débuts avait perdu de sa vigueur; le regard porté sur la $N R F$ se fit plus critique; Europe et Le Mercure de France occupaient la majeure partie

13. Cependant le prix ne fut décerné qu'une seule fois. 
des comptes rendus de périodiques français; Proust et Valéry essuyèrent des attaques répétées, non d'Eliot lui-même, assurément, mais de ses collaborateurs (Richard Aldington et F. S. Flint, en particulier). Il est surtout un signe qui ne trompe pas sur la perte générale d'influence de la revue de Gallimard : à l'exception de Fernandez et Cocteau, les auteurs de la maison y devinrent plus rares; Charles Mauron et Jacques Maritain opérèrent une entrée en force; mais, plus significativement encore, deux ennemis de la revue furent généreusement accueillis : Henri Massis et Maurras. Toutes ces transformations eurent lieu à partir de 1926, à peu près contemporaines donc à la fois de la dernière chronique d'Eliot à la $N R F$ et de sa double conversion à l'anglocatholicisme et au royalisme. La distance prise soudain avec La Nouvelle Revue française paraît ainsi devoir être mise en rapport avec le tournant politique et religieux de l'écrivain, tournant qui allait s'exprimer de plus en plus clairement dans ses écrits comme dans The Criterion. L'incompatibilité devait nécessairement se manifester, entre un auteur de plus en plus influencé par l'Action française et une revue qui avait affirmé depuis toujours son opposition au mouvement maurrassien, et de façon encore plus évidente après la condamnation vaticane de 1926.

Cette incompatibilité datait de la création même du Criterion. En 1919, par réaction aux années de guerre qui avaient précédé, Rivière avait expressément voulu faire de la $N R F$ l'organe d'une littérature pure, débarrassée de toute préoccupation autre qu'esthétique, en défendant avec véhémence « une certaine gratuité » de l'activité artistique ${ }^{14}$. Eliot proposait au contraire un programme ambigu : tout en reconnaissant une certaine autonomie de la littérature, il affirmait de façon non moins pressante une solidarité de l'activité littéraire avec les autres activités sociales et, en particulier, la politique, dont la revue se devait aussi de rendre compte ${ }^{15}$. C'est dire que, dès l'origine du Criterion, rien n'interdisait un engagement politique qui se produisit en effet quelques années plus tard. Ainsi, au moment même où Eliot commençait à collaborer avec la $N R F$, se précisaient les premiers symptômes d'une divergence à venir.

\section{Un différend esthétique}

Cette ambivalence politique se doubla toutefois d'un véritable différend esthétique. Celui-ci ne s'exprima sans doute jamais plus nettement que dans l'article de Rivière sur " La Crise du concept de littérature », en 1924. Eliot en fit immédiatement l'éloge, en approuvant la façon dont Rivière dénonçait la confusion de la littérature avec la religion ${ }^{16}$. Mais cette thèse, bien propre à recueillir l'approbation du futur auteur de The Use of Poetry and the Use

14. Jacques Rivière, «La Nouvelle Revue Française », La Nouvelle Revue française, $\mathrm{n}^{\circ} 69$, juin 1919 , p. 31.

15. T. S. Eliot, « The Function of a Literary Review », Criterion, ${ }^{\circ} 4$, juillet 1923, p. 421.

16. Id., «A Commentary », Criterion, $\mathrm{n}^{\circ}$ 8, juillet 1924, p. 373. 
of Criticism ${ }^{17}$, lui cachait une divergence profonde. Eliot aurait dû repérer la violente condamnation lancée par Rivière contre les effets de l'« esprit moderne » et du « surréalisme » dans les arts, qu'il résumait par la notion de « catalyse » :

Non plus seulement l'idée de littérature, mais aussi celle de peinture, de musique, peut-être même de poésie disparaissent devant celle, si l'on veut, de catalyse, le moi créateur n'étant plus qu'un corps qui par sa seule présence et sans y participer chimiquement met en jeu les affinités de deux corps étrangers ${ }^{18}$.

L'image de la catalyse venait chez Rivière connoter négativement un processus artistique où le créateur cherche à exprimer son expérience dans un matériau (langage, peinture, sons), sans intervenir dans la transposition et en s'efforçant de court-circuiter toute médiation et tout contrôle par le moi. L'écriture surréaliste était clairement visée.

Or, l'image de la catalyse était précisément celle qu'avait utilisée Eliot dans l'un de ses essais les plus célèbres, "Tradition and the Individual Talent », pour représenter la création poétique ${ }^{19}$. Il est peu probable que Rivière ait eu connaissance de ce texte. La comparaison y était développée dans des proportions autrement plus considérables, mais avec une signification grosso modo identique. Une seule différence, essentielle, séparait les deux textes : la catalyse éliotienne était connotée positivement, et non pas négativement comme chez Rivière. C'est donc bien sur le fond que portait la divergence. Comment s'en étonner, puisque Eliot faisait l'apologie de cette même impersonnalité de l'art que Rivière condamnait absolument? Au-delà de cette théorie de l'impersonnalité, c'est toute l'esthétique moderniste qui était en jeu, revendiquée et théorisée par l'un, rejetée par l'autre. Retenons de ce malentendu qu'une même revendication de classicisme conduisait les deux critiques à des prises de position opposées : au nom de l'esthétique classique, Rivière, d'un côté, condamnait la création artistique la plus innovante tandis qu'Eliot, de son côté, la soutenait. C'est que peut-être ils ne se référaient pas exactement au même classicisme ni au même modernisme.

Ce différend esthétique explique sans doute une absence de taille dans la collaboration d'Eliot avec la $N R F$ : la poésie. Déjà, la première fois que Gide avait écrit à l'écrivain, il laissait entendre qu'il ne le connaissait que comme l'auteur de The Sacred Wood; piqué au vif, Eliot promit de lui envoyer ses

17. Id., The Use of Poetry and the Use of Criticism (1933), Londres, Faber and Faber, 1964, p. 113-114.

18. Jacques Rivière, «La Crise du concept de littérature », La Nouvelle Revue française, $\mathrm{n}^{\circ} 125$, février 1924, p. 399.

19. T. S. Eliot, Selected Essays, op. cit., p. 17-19. Pour une analyse détaillée de cette image, voir William Marx, Naissance de la critique moderne : la littérature selon Eliot et Valéry (1889-1945), Arras, Artois Presses Université, 2002, p. 202-206. 
poésies, qui lui semblaient « au moins supérieures à [ses] proses $^{20} »$. Encore, en 1921, Eliot n'avait-il pas publié The Waste Land, qui allait bouleverser le paysage littéraire anglophone. Mais de cette déflagration moderniste on ne perçut nul écho dans la $N R F$. C'est la revue Commerce qui eut le privilège de faire découvrir au public français la poésie d'Eliot en 1924, suivie peu après du Navire d'Argent; et The Waste Land parut pour la première fois en français dans Esprit en 1926. Plus étonnant encore : quand en 1925 Fernandez publia dans la $N R F$ un article sur son ami d'outre-Manche, il passa entièrement sous silence son œuvre poétique. À une omission si paradoxale il y avait peut-être une raison toute simple : la poésie d'Eliot était beaucoup plus novatrice, voire révolutionnaire, que ne le laissait attendre sa critique; elle avait aussi peu droit de cité dans le cadre esthétique en partie néoclassicisant de la $N R F$ de l'immédiat après-guerre que, de son côté, l'Ulysse de Joyce.

Se dessine ainsi une double face de la $N R F$ à cette époque : si les articles critiques pouvaient manifester souvent le désir d'un renouvellement de la littérature, avec d'autant plus de liberté qu'ils traitaient de littérature étrangère, comme sous la plume de Larbaud, et qu'ils étaient classés dans la rubrique " Notes », en revanche la place d'honneur de la revue, c'està-dire la partie proprement dévolue à la création, demeurait beaucoup plus fermée aux innovations. Il y avait plus d'audace dans les mots et les programmes que dans les faits. Ce double discours procédait-il d'une réelle tiédeur des convictions esthétiques ou d'une stratégie commerciale imposée par l'éditeur, qui cherchait à ne pas exagérément choquer les abonnés? Probablement toutes ces raisons à la fois, à quoi il faudrait sans doute ajouter la volonté de préserver dans la revue une certaine polyphonie démocratique de bon aloi. Eliot semblait bien conscient du problème lorsqu'en 1924 il expliquait la faiblesse du mouvement classique en France par sa préférence pour la critique plutôt que pour la création ${ }^{21}$; le reproche paraissait viser autant la $N R F$ que les littérateurs gravitant autour de l'Action française.

Toutefois cette explication ne laisse pas de faire problème : n'est-il pas paradoxal, en effet, que ce soit justement quand Eliot revendiqua avec le plus de force la référence au classicisme, c'est-à-dire autour de 1927, qu'il se soit éloigné d'une revue pour qui le classicisme avait servi de référence permanente, au moins depuis la reprise en main par Rivière en 1919? Si contradiction il y a, elle n'est en fait qu'apparente : le terme de classicisme n'avait pas nécessairement le même sens pour les deux parties.

\section{Cultures à classiques, cultures à tradition et à canon}

En 1928, Eliot décrivait ainsi sa doctrine générale : «Classicist in literature, royalist in politics, and anglo-catholic in religion ${ }^{22} . »$ Ces trois termes

20. T. S. Eliot, Letters, op. cit., p. 494.

21. Id., "A Commentary », Criterion, $\mathrm{n}^{\circ}$ 7, avril 1924, p. 231.

22. Id., For Lancelot Andrewes : Essays on Style and Order, Londres, Faber \& Gwyer, 
correspondaient exactement à ceux qu'en 1913, dans deux longues études parues dans la $N R F$, Thibaudet nommait « L'esthétique des trois traditions » : «Classique, catholique, monarchique ${ }^{23}$.» Eliot eut-il connaissance de cet article? C'est probable : de retour à Harvard à cette époque, Eliot s'était abonné à la revue. En tout cas, sa profession de foi de 1928 s'opposait frontalement aux thèses de Thibaudet, qui ne citait ces trois traditions que pour les rejeter et pour récuser, en particulier, l'espoir maurrassien d'une renaissance classique, c'est-à-dire la possibilité d'une résurrection littérale du classicisme : « Je crois bien que l'on peut poser ce principe, affirmait-il, qu'une littérature classique ne saurait naître d'une autre dans la même langue ${ }^{24}$. \ De la même manière, dans son article programmatique de 1919, Rivière prenait bien soin de préciser que la « renaissance classique » qu'il prévoyait ne saurait être « textuelle et de pure imitation, comme les disciples de Moréas et les écrivains de La Revue critique l'entendaient et la définissaient avant la guerre, mais profonde et intérieure $»$; Rivière ne parlait de classicisme que par analogie. Entre Thibaudet et Rivière, de l'avant à l'après-guerre, on discerne donc une relative continuité de la réflexion de la $N R F$ sur le classicisme.

Une seule renaissance classique littérale restait possible néanmoins, selon Thibaudet : celle d' " un classicisme étranger s'inspirant du classicisme français ${ }^{25} »$, de sorte que l'impossibilité d'une renaissance classique en France ne valait pas nécessairement pour l'étranger. Autrement dit, Thibaudet s'insurgeait non pas contre l'idée même de classicisme, mais contre ce qu'il appelait «les doctrines de tradition », s'érigeant en gardiennes de conceptions figées. À bien des égards, l'esthétique éliotienne ne pouvait tomber sous le coup d'une telle condamnation, puisqu'elle consistait justement à promouvoir dans la littérature de langue anglaise un classicisme d'inspiration française.

Pourtant, il est significatif que, dans les pages de la $N R F$, Fernandez ait reproché à Eliot son traditionalisme. Dans son article consacré au « classicisme de T. S. Eliot ${ }^{26} »$, il accusait le critique anglo-américain de défendre une vision trop rigide du classicisme, ne prenant pas assez en compte la situation particulière où se trouve chaque écrivain : selon Fernandez, le classicisme ne pouvait être que de nature relative, et non point absolue ${ }^{27}$,

1928, p. 9.

23. Albert Thibaudet, «L'esthétique des trois traditions », La Nouvelle Revue française, $\mathrm{n}^{\circ} 51$, mars 1913, p. 355.

24. Ibid., p. 374.

25. Ibid., p. 377.

26. Ramon Fernandez, «Le Classicisme de T.S. Eliot», La Nouvelle Revue française, $\mathrm{n}^{\circ} 137$, février 1925 , p. $246-251$.

27. Sur le fond, on pourrait contester cette interprétation absolutiste de la doctrine éliotienne : la réévaluation de Baudelaire et de Laforgue démontrait au contraire chez Eliot un véritable sens du relativisme (voir William Marx, Naissance de la critique moderne, op. cit., p. 87-95, 267-270). 
alors que le classicisme défendu par Eliot apparaissait au contraire comme une « doctrine de tradition » telle que l'avait définie Thibaudet, empreinte d'une raideur excessive.

Quelle situation étrange, où deux interlocuteurs se réclament du classicisme, mais ne parviennent pas à s'entendre sur le terme ! C'est qu'en réalité ils ne parlent pas de la même chose. On se trouve en plein dans un malentendu culturel, imputable au fait que la notion de classicisme, si prégnante en France, est à peu près absente de la culture anglo-saxonne, où prévaut plutôt celle de tradition et de canon, développée à partir du $\mathrm{XIX}^{\mathrm{e}}$ siècle quand, sous l'influence protestante, il s'est agi de structurer la culture séculière sur le modèle du canon de la Bible. Or, les deux concepts, si voisins qu'ils puissent paraître, ne sont pas superposables, de sorte qu'il est possible de retrouver sous les termes de tradition, de canon et de classiques une spécialisation latente, comme autant de versions nationales et culturelles d'un même concept global. Dans chaque cas, il s'agit de désigner une valeur esthétique de référence, mais cette valeur ne fonctionne pas de la même manière, selon qu'elle est nommée classicisme d'un côté, tradition ou canon de l'autre.

Car classiques et canon n'évoluent pas selon le même processus. Cette dichotomie est parallèle à l'opposition entre droit romain et droit coutumier (common law) : alors que le premier définit abstraitement une règle générale, le second ne connaît que des cas particuliers et concrets. De même, alors que le classique s'exprime comme un tout, le canon détaille et dresse une liste. Dans les cultures à classiques, le classique est le plus souvent implicite, il relève du non-dit; dans les cultures à tradition, la tradition fait toujours débat. On ne peut échapper au classique qu'en le refusant d'un bloc; on échappe à la tradition en la transformant, tout simplement, et en proposant un nouveau canon.

C'est pourquoi la culture française et plus généralement latine des classiques travaille sur le mode de l'alternative radicale, dont la Révolution ou bien l'opposition binaire avec le romantisme fournissent le modèle; l'évolution se produit par ruptures violentes, suivies d'une intégration tardive; le moyen d'expression critique privilégié est le débat théorique et le manifeste. La culture anglo-saxonne de la tradition ou du canon pratique au contraire la refonte permanente : le canon est en perpétuelle transformation, plus ou moins progressive, intégrant les innovations tout en recomposant à mesure le passé; l'évolution se produit par restructurations et développements rapides; le débat critique minimise la théorie et se concentre sur une réécriture pragmatique du canon.

Ainsi, en France, Dada et le surréalisme refusent-ils tout héritage, à part celui de quelques rares précurseurs, auxquels ils se sentent de toute façon supérieurs; ils sont anticlassiques par principe; mais par là, malgré eux, ils participent déjà de la culture classique. Le modernisme anglo-américain, au contraire, est traditionnel et canonique; il fait de la réforme du canon le 
levier irremplaçable de la rénovation littéraire; il tente d'imposer ce canon nouveau de manière autoritaire, mais manifeste à son égard la plus grande déférence, dénuée de toute condescendance. Eliot et Pound n'agirent pas autrement : ils recomposèrent leur héritage littéraire, en excluant, d'un côté, les poètes romantiques et en réévaluant, de l'autre, la poésie courtoise occitane du Moyen Âge et la poésie métaphysique du XVII ${ }^{\mathrm{e}}$ siècle, notamment.

Lorsque le critique Eliot employait le terme de classicisme, il subissait une influence française, à l'évidence. Mais, sur le fond, il concevait ce classicisme de manière anglo-saxonne, comme un outil de transformation de la littérature. Son vocabulaire critique faisait donc paraitre Eliot pour plus français qu'il n'était en réalité. Malgré les apparences, son classicisme autoritaire, avec lequel Fernandez ne se sentait pas en accord, fonctionnait en fait sur le mode du canon anglophone, et rejoignait étrangement le classicisme des néoclassiques, de Maurras ou de Hulme, c'est-à-dire une forme aberrante du classicisme à la française, et d'autant plus atypique que ses partisans se réclamaient non de la référence classique française habituelle, mais de l'image repoussoir du classicisme qu'avait créée le romantisme pour s'y opposer: un classicisme dictatorial et antilibéral. Ainsi, par un nouveau malentendu, le rapprochement d'Eliot avec l'Action française était inévitable. Au contraire, pour la $N R F$, fidèle à un classicisme réel et non pas arbitrairement reconstruit, la notion de classicisme agissait comme une référence diffuse et floue, comme un opérateur de conciliation, n'excluant les avant-gardes que parce que celles-ci, en France, rejettent nécessairement le passé.

Aussi la revendication éliotienne du classicisme se prêtait-elle à tous les malentendus de l'autre côté de la Manche : les rédacteurs français de la $N R F$ ne savaient pas comment concilier l'apparent classicisme autoritaire du critique Eliot avec le radicalisme moderniste du poète, qu'ils percevaient, selon les critères français, comme un anticlassicisme, alors qu'ils auraient dû le comprendre à la manière anglaise, comme un nouveau développement de la tradition et du canon. Ce qui paraissait cohérent dans le monde anglophone apparaissait comme une contradiction en France. C'est qu'à la différence du modernisme anglo-américain, qui naît dans une culture de tradition, souple et mobile, le modernisme à la française, issu au contraire d'une culture à classiques, stables et figés, ne peut lui-même se penser que comme anticlassique, quitte à devenir un classique à son tour bien des années plus tard, lorsqu'une nouvelle révolution esthétique l'aura fait rentrer dans l'histoire. Il y a deux modernismes parce qu'il y a deux façons de se relier au passé, par la rupture ou par la continuité. Voilà ce qu'Eliot apprit à ses dépens au contact de La Nouvelle Revue française.

William Marx Université Paris Ouest-Institut universitaire de France 